\title{
Relationships between radiological pattern and cell-mediated immune response in Mycoplasma pneumoniae pneumonia
}

\author{
H. Tanaka, H. Koba, S. Honma, F. Sugaya, S. Abe
}

Relationships between radiological pattern and cell-mediated immune response in Mycoplasma pneumoniae pneumonia. H. Tanaka, H. Koba, S. Honma, F. Sugaya, S. Abe. CERS Journals Ltd 1996.

ABSTRACT: The aim of this study was to determine the relationship between the radiological pattern of Mycoplasma pneumoniae and the level of cell-mediated immunity of the host.

Computed tomographic (CT) scans of the chest and the results of the purified protein derivative (PPD) test were studied during the acute stage of infection in 54 patients with $M$. pneumoniae pneumonia. The CT findings were used to divide the patients into two groups: one group had a predominance of nodular opacities with a centrilobular distribution (Group $\mathrm{N} ; \mathrm{n}=29$ ); and the other showed a predominance of an airspace consolidation (Group $C ; n=25$ ).

Forty out of 54 subjects had negative tuberculin skin tests $(<10 \mathrm{~mm}$ induration). The positive rate of PPD reaction was higher in Group $N$ (13 out of 29) compared to Group $\mathrm{C}$ ( 1 out of 25 ) ( $\mathrm{p}=\mathbf{0 . 0 0 0 5}$ ); whilst pleural effusion appeared more frequently in Group $\mathrm{C}(10$ out of 25$)$ than in Group $\mathrm{N}(3$ out of 29$)(\mathrm{p}=\mathbf{0 . 0 2 3})$. There was no significant difference between Groups $N$ and $C$ in white blood cell and lymphocyte counts, level of antibodies to $M$. pneumoniae in sera, and severity of the disease.

These findings suggest that the characteristics of the host cell-mediated immunity might influence the pattern of pulmonary lesions in $M$. pneumoniae infection. Eur Respir J., 1996, 9, 669-672.

\author{
Third Department of Internal Medicine, \\ Sapporo Medical University School of \\ Medicine, Sapporo, Japan.
}

Correspondence: H. Tanaka

Third Department of Internal Medicine Sapporo Medical University

South-1, West-16

Chuo-ku

Sapporo 060

Japan

Keywords: Cell-mediated immunity computed tomography

Mycoplasma pneumonia

purified protein derivative

tuberculin anergy

Received: April 101995

Accepted after revision November 41995
The cell-mediated immunity (CMI) of the host plays an important role in the development of Mycoplasma pneumoniae pneumonia (MP) [1,2]. Mizutani et al. [3] reported that the delayed hypersensitivity skin reactions to $M$. pneumoniae antigen appeared to correlate with severity of pneumonia in human MP. Foy et al. [4] reported that MP infection in patients with immunodeficiency syndrome had a lack of radiological chest findings. Putman et al. [5] reported a bilateral reticulonodular pattern when MP was associated with sarcoidosis. These manifestations suggest that pulmonary infiltrates of MP might be a result of the immunological reaction of the host.

The purified protein derivative (PPD) reaction is used to confirm past infection by Mycobacterium tuberculosis, and to determine the CMI of the host. In Japan, most individuals have CMI to PPD due to the nearly universal bacille Calmette-Guérin (BCG) vaccination in childhood. Transient tuberculin anergy has been observed $(57-61 \%)[6,7]$ during the early stage of MP. TsunEKAwA et al. [7] reported that blastogenic lymphocyte response to PPD and PPD-induced gamma-interferon (IFN- $\gamma$ ) production were significantly reduced in tuberculin-negative patients with MP. However, the relationship between the pattern of pulmonary lesions and host CMI level in MP has not been evaluated.

The findings of chest radiography of MP are varied; CLYDE [8] noted four frequent patterns suggesting MP: bronchopneumonia; nodular infiltration; plate-like atelectasis; and hilar adenopathy. Bronchopneumonia of MP is accompanied by thickening of bronchi, streaks of interstitial infiltration, and small areas of subsegmental atelectasis. The changes are produced by the presence of peribronchial inflammatory cell infiltration and intraluminal exudates that may obstruct bronchioles [9]. Standard (CT) and high-resolution computed tomography (HRCT) are more useful than chest roentgenography in evaluating the interstitial pulmonary changes, typical of subtle lung disease and emphysema [10], because many parenchymal structures and interstitial changes are superimposed on the chest roentgenogram. CT analysis of MP seems to be much more useful than posteroanterior chest roentgenogram [11].

This study was designed to search for an association between the radiological pattern and the host CMI in patients with MP. A comparison was made between the CT patterns and the size of PPD skin test during the acute stage of $M$. pneumoniae infection.

\section{Methods}

\section{Study population}

Fifty four patients (23 males and 31 females) with MP, who visited Sapporo Medical University Hospital or 
one of its branch hospitals over the period 1986-1992, were enrolled. All patients with community-acquired acute pneumonia who underwent CT scans and tuberculin skin test on the same day within 7 days from the onset of symptoms were initially selected, excluding those with the following conditions: 1) purulent sputum;2) underlying pulmonary disease, including chronic bronchitis, bronchiectasis, diffuse panbronchiolitis, or pulmonary emphysema; 3) pulmonary tuberculosis; 4) treatment with antibiotics before the hospital visit; 5) treatment with corticoids or immunosuppressants for other diseases; or 6) a recent negative PPD skin reaction. Participants who had serologically established MP were finally enrolled in this study. The age range was 15-67 years (mean 33 yrs).

The diagnosis of $M$. pneumoniae infection was confirmed by a fourfold or greater rise in the indirect haemagglutination (IHA) titre between acute and convalescent stages, or an IHA titre of $\geq 320$ in convalescent stage [9, 12]. This titre was assessed using a commercial test kit (Serodia-Myco II, Fujirebio Inc., Japan).

\section{Computed tomography}

CT scan was performed within 7 days from the onset of symptoms. It was obtained on a CT scanner (GE9800 , General Electric or TCT-60, Toshiba) using 5 or $10 \mathrm{~mm}$ collimation at $10 \mathrm{~mm}$ intervals, at full inspiration in the supine position. CT scans were interpreted by two thoracic radiologists without knowledge of the clinical data. The major CT manifestations were nodular opacities having centrilobular distribution (fig. 1), and consolidation with an air bronchogram (fig. 2).

The severity of the nodular opacities and of the consolidation in all five lobes was scored as either 0,1 or 2 , with 2 being the most severe. The nodular scores (NS) for all lobes and the consolidation scores (CS) for all lobes were each summed to get total nodular scores and total consolidation scores, and from these, the patients were divided into two groups. When the NS was higher than the CS, the patient was enrolled in the nodular opacities predominant group (Group $\mathrm{N}$; $\mathrm{n}=29$ ),

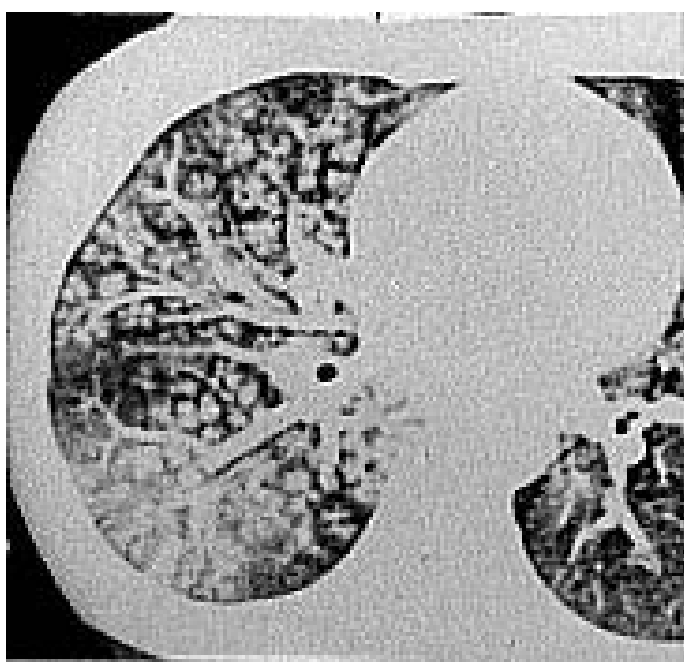

Fig. 1. - Typical computed tomography (CT) finding of nodular opacities having a centrilobular distribution. Nodular densities are located at the ends of bronchioles and pulmonary arteries (Group N). Group N: patients with nodular score higher than consolidation score.

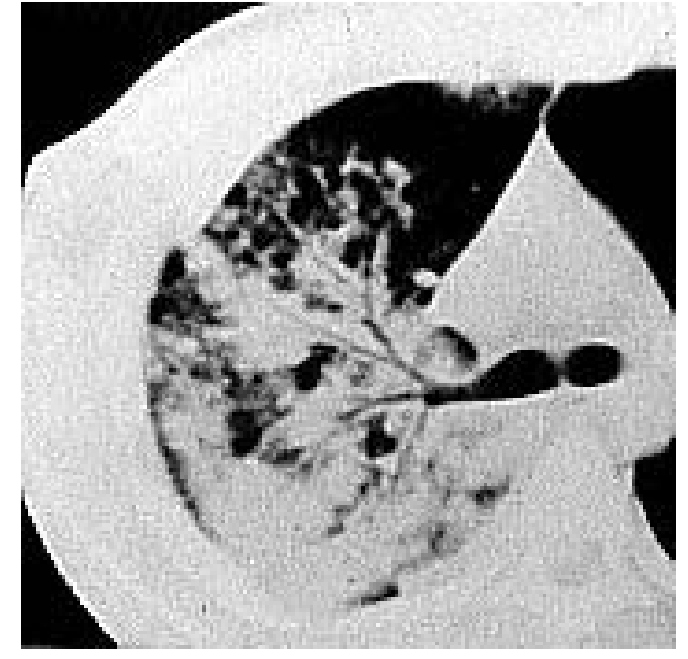

Fig. 2. - Typical computed tomographic (CT) appearance of consolidation with an air-bronchogram (group C). Group C: patients with consolidation score higher than nodular score.

and when the CS was higher than the NS, the patient was enrolled in the consolidation predominant group (Group $\mathrm{C} ; \mathrm{n}=25$ ).

The severity of the disease was determined according to previous studies $[13,14]$. Cases with hypoxaemia (arterial oxygen tension $\left(\mathrm{Pa}_{\mathrm{a}} \mathrm{O}_{2}\right)<9.3 \mathrm{kPa}(<70$ torr) or who needed more than 14 days of hospitalization were considered as having severe clinical courses. There were no significant differences between groups $\mathrm{N}$ and $\mathrm{C}$ as regards age, gender, peripheral white blood cell or lymphocyte counts (table 1).

\section{$P P D$ skin reaction}

Patients were injected intracutaneously on the forearm with $0.05 \mu \mathrm{g} \cdot 0.1 \mathrm{~mL}^{-1}$ of PPD on the same day as the CT scan. The skin test was read after $48 \mathrm{~h}$. An induration of $<10 \mathrm{~mm}$ in diameter was considered negative and one of $\geq 10 \mathrm{~mm}$ was considered positive [15].

\section{Statistical analysis}

All statistical analyses were performed on a Macintosh computer using a Statview 4.0 program (Brainpower, Calabases, CA, USA). The $\mathrm{N}$ and $\mathrm{C}$ groups were compared in terms of age, peripheral white blood cell and lymphocyte counts, number of affected pulmonary lobes in CT, and sera M. pneumoniae titre. Data were Table 1. - Characteristics of patients with Mycoplasma pneumoniae infection

\begin{tabular}{lccc}
\hline & $\begin{array}{c}\text { Group N } \\
(\mathrm{n}=29)\end{array}$ & $\begin{array}{c}\text { Group C } \\
(\mathrm{n}=25)\end{array}$ & $\mathrm{p}$-value \\
\hline Age yrs & $35 \pm 13$ & $30 \pm 11$ & $\mathrm{NS}$ \\
Sex M/F & $13 / 16$ & $10 / 15$ & NS \\
WBC cells $\times 10^{9} \cdot \mathrm{L}^{-1}$ & $7.0 \pm 2.6$ & $7.7 \pm 3.0$ & NS \\
Lymphocyte cells $\times 10^{9} \cdot \mathrm{L}^{-1}$ & $1.6 \pm 8.7$ & $1.4 \pm 0.78$ & $\mathrm{NS}$ \\
\hline
\end{tabular}

M: male; F: female; WBC: white blood cell count; ns: nonsignificant. Group N: patients with nodular score higher than consolidation score; Group C: patients with consolidation score higher than nodular score. 
expressed as mean \pm SD and the Mann-Whitney U-test was used to compare the quantitative variables studied. The groups were also compared for PPD positive rate, gender, disease severity, mediastinal lymph node swelling and pleural effusion on CT, using the Chi-squared test. A p-value of less than 0.05 was required for statistical significance.

\section{Results}

The results of the severity of the disease and CT manifestations are summarized in table 2. There was no significant difference in disease severity; however, Group $\mathrm{C}$ had a few more severe cases than Group N (3 out of 29 vs 7 out of 25). Pleural effusion was present in 13 out of 54 cases on CT; the effusion appeared bilaterally in five, on the right side in five, and on the left side in three. Pleural effusion could be detected in only 6 out of 13 cases when using posteroanterior and lateral chest roentgenograms. Pleural effusion was observed significantly more often in Group C than in Group N (10 out of 25 vs 3 out of 29; $\mathrm{p}=0.023$ ). There was no significant difference in the number of affected pulmonary lobes and mediastinal lymph node enlargement when comparing both groups.

Of the 54 patients with MP, forty proved to be tuberculin-negative during the acute stage of infection. As shown in figure 3 , the rate of PPD-positive reaction $(\geq 10$ $\mathrm{mm}$ ) in Group $\mathrm{N}$ was significantly higher than that in Group C (13 out of 29 vs 1 out of $25 ; \mathrm{p}=0$. 0005). The Mann-Whitney U-test also indicated a significant difference $(p=0.024)$ between these two groups. Maximum values of serum IHA titre to $M$. pneumoniae during the clinical course are shown in figure 4 . There were no significant differences between Groups $\mathrm{N}$ and $\mathrm{C}$.

\section{Discussion}

In this study, we found that the rate of positive tuberculin test during the acute stage of MP was higher in patients with a chest CT scan indicating a predominance of a nodular type of pulmonary lesion than in those with a predominance of a consolidation pattern. This finding suggests that the varied radiological manifestations of MP might reflect the current host CMI level. PUTMAN

Table 2. - Disease severity and CT findings in mycoplasma pneumonia

\begin{tabular}{lccc}
\hline & $\begin{array}{c}\text { Group N } \\
(\mathrm{n}=29)\end{array}$ & $\begin{array}{c}\text { Group C } \\
(\mathrm{n}=25)\end{array}$ & p-value \\
\hline $\begin{array}{l}\text { Disease severity } \\
\text { Mild course }\end{array}$ & 26 & 18 & NS \\
$\begin{array}{l}\text { Severe course } \\
\text { CT findings }\end{array}$ & 3 & 7 & \\
$\begin{array}{l}\text { Number of affected lobes } \\
\text { Mediastinal LN swelling }\end{array}$ & $2.4 \pm 1.3$ & $2.1 \pm 1.2$ & NS \\
$\quad$ Yes & 14 & 11 & NS \\
$\quad$ No & 15 & 14 & \\
$\begin{array}{l}\text { Pleural effusion } \\
\quad \text { Yes }\end{array}$ & 3 & 10 & 0.023 \\
$\quad$ No & 26 & 15 & \\
\hline
\end{tabular}

LN: lymph node; CT: computed tomography; NS: nonsignificant. For definition of groups see legend to table 1.

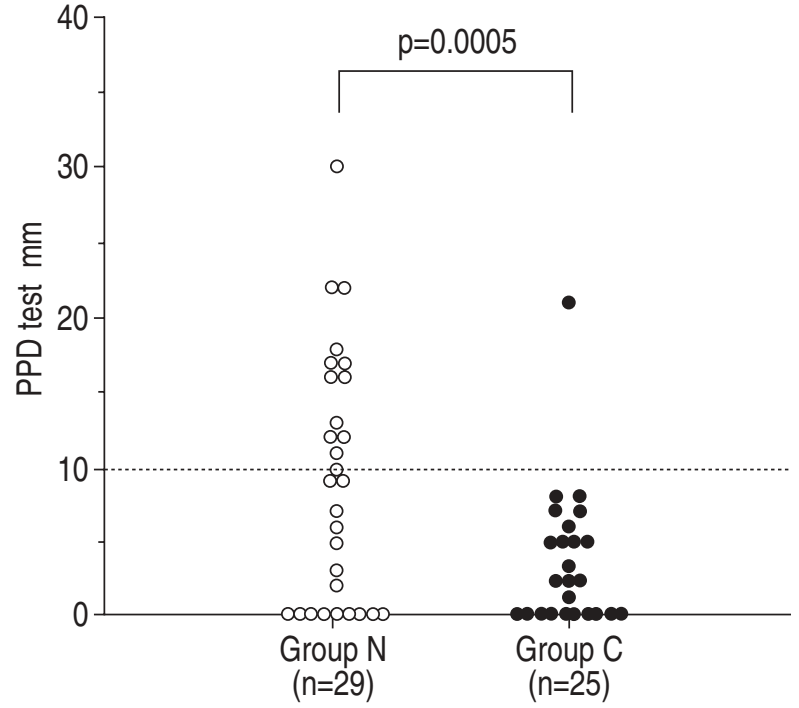

Fig. 3. - Distribution of PPD results in millimetres of induration within 7 days of onset of mycoplasma pneumonia. The positive rate in Group $\mathrm{N}$ is significantly higher $(\mathrm{p}=0.0005)$ than that in Group $\mathrm{C}$, using the Chi-squared test. PPD: purified protein derivative (tuberculin). For definition of Groups $\mathrm{N}$ and $\mathrm{C}$ see legend to figures 1 and 2.

et al. [5] reported that the radiographic appearance of MP in sarcoidosis showed a bilateral reticulonodular pattern. As the bronchoalveolar lavage (BAL) of sarcoidosis is characterized by an increase of lymphocytes and an elevated CD4/CD8 ratio [16], it is possible that the heightened CMI at the sites of pulmonary lesions might explain why the occurrence of mycoplasma infection produced a nodular pattern in the lung.

The radiological features of MP are variable, including bronchopneumonia, plate-like atelectasis, nodular infiltration and a streaking pattern outward from the hilum [5, $8,9]$, and are indistinguishable from bacterial or viral pneumonia [17]. We could not clearly divide the chest radiographs of our patients into the above-mentioned patterns because of the variety of findings. PUTMAN et al. [5] reported a study of 100 patients and recognized two roentgraphic patterns using conventional chest roentgenogram:

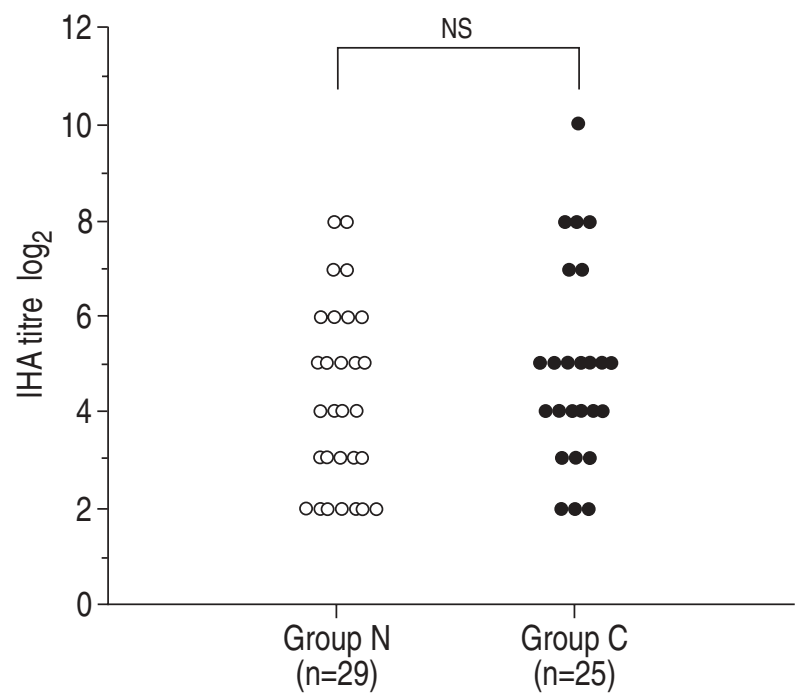

Fig. 4. - Distribution of maximum values of serum indirect haemagglutination (IHA) titre of $M$. pnuemoniae during the clinical course. There is no significant difference (NS) between the groups. For definition of Groups $\mathrm{N}$ and $\mathrm{C}$ see legend to figures 1 and 2 . 
the first group had segmental or lobar consolidation with an air bronchogram (48\%); and the second group showed a bilateral reticulonodular pattern $(28 \%)$; however, this classification was crude. In contrast, CT findings could easily divide the cases into two groups using our classification; 13 patients showed only nodular opacity and four revealed only consolidation. The other 37 cases had mixed nodular opacity and consolidation patterns; however, the ratios of these two CT patterns were usually biased clearly toward one or the other group.

We could not obtain a lung biopsy specimen in this study. CLYDE [9] reported that the pathology of human MP shows peribronchial inflammatory cell infiltration and intraluminal exudates that may obstruct bronchioles. These pathological findings mimic those of a mouse mycoplasma model. We previously reported [18] the effect of interleukin-2 (IL-2) as a T-cell activator and cyclosporin A (CyA) as a T-cell selective suppressor on pulmonary lesions in such an animal model of mycoplasma infection. IL-2 treatment enhanced the level of CMI and, simultaneously, exacerbated peribronchial lymphocyte infiltration and accumulation of macrophages at the ends of bronchioles; these pathological lesions were mimicking nodular opacities on CT of human MP. In contrast, CyA treatment depressed CMI, reduced peribronchial lymphocyte infiltration and exacerbated intra-alveolar inflammatory cell accumulation; these pathological changes may correspond to airspace consolidation on CT of human MP [18]. These findings supported the suggestion that the pathological pattern of lung lesions in MP might reflect the level of host CMI.

We observed that 40 out of 54 patients had negative PPD reactions when tested in the acute phase of the infection. Previous reports have also indicated that 22 out of 36 [6], and 25 out of 44 [7] MP patients had a negative PPD reaction. TsunEKAWA et al. [7] reported that in PPD-negative MP patients the number of peripheral blood T-lymphocytes was not significantly lower, but blastogenic lymphocyte response to PPD and PPDinduced IFN- $\gamma$ production were significantly reduced compared with PPD-positive patients. LAGRANGE et al. [19] suggested that cutaneous tuberculin anergy could result if the number or activity of circulating committed T-cells was depressed, and cutaneous anergy has been demonstrated to result from circulating suppressor cell. Previous data support the role of the suppressor cells, monocytes or macrophages in impairing CMI reactivity and delayed hypersensitivity to PPD at sites distant from the active focus, without inhibiting the appropriate response in the area of inflammation $[15,20]$. These theories are supported by a study of lymphocyte subsets in peripheral blood and BAL in MP patients [21]; the number of CD4+ lymphocytes and the CD4/CD8 ratio was decreased in peripheral blood, whilst on the other hand, an increase of activated T-cell and an elevated CD4/CD8 ratio were found in BAL.

In conclusion, our study suggests that the CMI of patients with $M$. pneumoniae pneumonia might influence the radiographic pattern of the pulmonary lesions.

\section{References}

1. Fernald GW. Role of host response in Mycoplasma pneumoniae disease. J Infect Dis 1973; 127 (Suppl.): 55-58.
2. Biberfeld G, Biberfeld P, Sterner G. Cell-mediated immune response following Mycoplasma pneumoniae infection in man. I. Lymphocyte stimulation. Clin Exp Immunol 1974; 17: 29-41.

3. Mizutani H, Mizutani H, Kitayama N, et al. Delayed hypersensitivity in Mycoplasma pneumoniae infection. Lancet 1971; i: 186-187.

4. Foy HM, Ochs H, Davis SD, Kenney GE, Luce RR. Mycoplasma pneumoniae infections in patients with immunodeficiency syndromes: report of four case. $J$ Infect Dis 1973; 127: 388-393.

5. Putman CE, Curtis AM, Simeone JF, Jensen P. Mycoplasma pneumonia. Am J Roentgenol 1975: 124: 417422.

6. Biberfeld G, Sterner G. Tuberculin anergy in patients with Mycoplasma pneumoniae infection. Scand J Infect Dis 1976; 8: 71-73.

7. Tsunekawa H, Takagi E, Kishimoto H, Shimokata K. Depressed cellular immunity in Mycoplasma pneumoniae pneumonia. Eur J Respir Dis 1987; 70: 293-299.

8. Clyde WA Jr. Infections of the respiratory tract due to Mycoplasma pneumoniae. In: Chernick V, Kendig EL Jr, eds. Disorders of the Respiratory Tract in Children. 5th edn. Philadelphia, W.B. Saunders, 1990; pp. 403-412.

9. Clyde WA Jr. Clinical overview of typical Mycoplasma pneumoniae infections. Clin Infect Dis 1993; 17 (Suppl. 1): 32-36.

10. Naidich DP. Pulmonary parenchymal high-resolution CT. To be or not to be? Radiology 1987; 17: 22-24.

11. Tanaka H, Koba H, Mori T, et al. Computed tomographic study on Mycoplasma pneumoniae pneumonia. Jpn J Clin Radiol 1985; 30: 979-986.

12. Lind K. An indirect hemagglutination test for serum antibodies against Mycoplasma pneumoniae using formalinized, tanned sheep erythrocytes. Acta Pathol Microbiol Scand 1968; 73: 459-472.

13. Koletsky RJ, Weinstein AJ. Fulminant Mycoplasma pneumoniae infection. Am Rev Respir Dis 1980; 122: 491496.

14. Izumikawa K, Hara K. Clinical features of mycoplasma pneumonia in adults. Yale J Biol Med 1983; 56: 505510.

15. Pesanti EL. The negative tuberculin test: tuberculin, HIV and anergy panels. Am J Respir Crit Care Med 1994; 149: 1699-1709.

16. Hunninghake GW, Crystal RG. Pulmonary sarcoidosis: a disorder mediated by excess helper T-lymphocyte activity at sites of disease activity. N Engl J Med 1981; 305: 429-434.

17. Rosmus HH, Pare JAP, Masson AM, Fraser RG. Roentgenographic patterns of acute mycoplasma and viral pneumonia. J Can Assoc Radiol 1968; 19: 74-77.

18. Tanaka H, Yamagishi M, Abe S, Tamura H. The effect of interleukin-2 and cyclosporin A on the cell-mediated immunity in mycoplasma pneumonia of mice. Am J Respir Crit Care Med 1994; 149: A872.

19. LaGrange PH, Mackaness GB. Site of action of serum factors that block delayed-type hypersensitivity in mice. J Exp Med 1978; 148: 235-244.

20. Fujiwara H, Tsuyguchi I. Frequency of tuberculin-reactive T-lymphocytes in pleural fluid and blood from patients with tuberculous pleurisy. Chest 1986; 89: 530-532.

21. Hayashi S, Ichikawa Y, Fujino K, et al. Analysis of lymphocyte subsets in peripheral blood and bronchoalveolar lavage fluid in patients with pneumonia due to Mycoplasma pneumoniae. Jpn J Thoracic Dis 1986; 24: 162-167. 